Network Working Group

Request for Comments: 3649

S. Floyd

Category: Experimental

\title{
Highspeed TCP for Large Congestion Windows
}

Status of this Memo

This memo defines an Experimental Protocol for the Internet community. It does not specify an Internet standard of any kind. Discussion and suggestions for improvement are requested. Distribution of this memo is unlimited.

Copyright Notice

Copyright (C) The Internet Society (2003). All Rights Reserved.

Abstract

The proposals in this document are experimental. While they may be deployed in the current Internet, they do not represent a consensus that this is the best method for high-speed congestion control. In particular, we note that alternative experimental proposals are likely to be forthcoming, and it is not well understood how the proposals in this document will interact with such alternative proposals.

This document proposes Highspeed TCP, a modification to TCP's congestion control mechanism for use with TCP connections with large congestion windows. The congestion control mechanisms of the current Standard TCP constrains the congestion windows that can be achieved by TCP in realistic environments. For example, for a standard TCP connection with 1500-byte packets and a $100 \mathrm{~ms}$ round-trip time, achieving a steady-state throughput of 10 Gbps would require an average congestion window of 83,333 segments, and a packet drop rate of at most one congestion event every 5,000,000,000 packets (or equivalently, at most one congestion event every $12 / 3$ hours). This is widely acknowledged as an unrealistic constraint. To address this limitation of TCP, this document proposes Highspeed TCP, and solicits experimentation and feedback from the wider community. 
Table of Contents

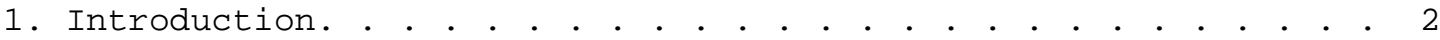

2. The Problem Description.. . . . . . . . . . . . . . . . . 3

3. Design Guidelines.. . . . . . . . . . . . . . . . . . . . . . . 4

4. Non-Goals.. . . . . . . . . . . . . . . . . . . . . . 5

5. Modifying the TCP Response Function.. . . . . . . . . . . . . . 6

6. Fairness Implications of the Highspeed Response

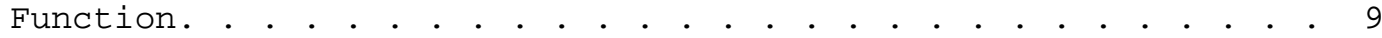

7. Translating the Highspeed Response Function into Congestion Control Parameters . . . . . . . . . . . . . . . 12

8. An alternate, linear response functions.. . . . . . . . . . . . 13

9. Tradeoffs for Choosing Congestion Control Parameters. . . . . 16 9.1. The Number of Round-Trip Times between Loss Events . . . 17 9.2. The Number of Packet Drops per Loss Event, with Drop-Tail. 17

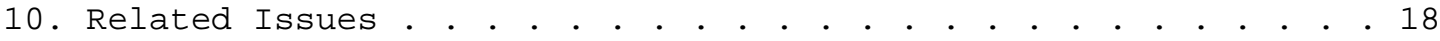
10.1. Slow-Start. . . . . . . . . . . . . . . . . . 18 10.2. Limiting burstiness on short time scales. . . . . . . . 19 10.3. Other limitations on window size. . . . . . . . . . . . . 19 10.4. Implementation issues.. . . . . . . . . . . . . . . 19

11. Deployment issues. . . . . . . . . . . . . . • . • . . . . 20 11.1. Deployment issues of Highspeed TCP. . . . . . . . . . . . 20 11.2. Deployment issues of Scalable TCP . . . . . . . . . . . 22

12. Related Work in HighSpeed TCP. . . . . . . . . . . . . . . 23

13. Relationship to other Work. . . . . . . . . . . . . . . 25

14. Conclusions. . . . . . . . . . . . . . . . . . . . . . . 25

15. Acknowledgements . . . . . . . . . . . . . . . . . . 25

16. Normative References . . . . . . . . . . . . . . . 26

17. Informative References . . . . . . . . . . . . . . 26

18. Security Considerations. . . . . . . . . . . . . . . . . 28

19. IANA Considerations. . . . . . . . . . . . . . . . . . . 28

A. TCP's Loss Event Rate in Steady-State. . . . . . . . . . . 29

B. A table for $\mathrm{a}(\mathrm{w})$ and $\mathrm{b}(\mathrm{w})$. . . . . . . . . . . . . . . . 30

C. Exploring the time to converge to fairness . • • • • • • • • 32 Author's Address . . . . . . . . . . . . . . . . . . 33 Full Copyright Statement . . . . . . . . . . . . . . . . . 34

\section{Introduction}

This document proposes Highspeed TCP, a modification to TCP's congestion control mechanism for use with TCP connections with large congestion windows. In a steady-state environment, with a packet loss rate $\mathrm{p}$, the current standard TCP's average congestion window is roughly 1.2 /sqrt (p) segments. This places a serious constraint on the congestion windows that can be achieved by TCP in realistic environments. For example, for a standard TCP connection with 1500byte packets and a $100 \mathrm{~ms}$ round-trip time, achieving a steady-state throughput of $10 \mathrm{Gbps}$ would require an average congestion window of 
83,333 segments, and a packet drop rate of at most one congestion event every 5,000,000,000 packets (or equivalently, at most one congestion event every $12 / 3$ hours). The average packet drop rate of at most $2 * 10^{\wedge}(-10)$ needed for full link utilization in this environment corresponds to a bit error rate of at most $2^{\star} 10^{\wedge}(-14)$, and this is an unrealistic requirement for current networks.

To address this fundamental limitation of TCP and of the TCP response function (the function mapping the steady-state packet drop rate to $\mathrm{TCP}^{\prime} \mathrm{s}$ average sending rate in packets per round-trip time), this document describes a modified TCP response function for regimes with higher congestion windows. This document also solicits experimentation and feedback on Highspeed TCP from the wider community.

Because Highspeed $\mathrm{TCP}^{\prime} \mathrm{S}$ modified response function would only take effect with higher congestion windows, Highspeed TCP does not modify TCP behavior in environments with heavy congestion, and therefore does not introduce any new dangers of congestion collapse. However, if relative fairness between Highspeed TCP connections is to be preserved, then in our view any modification to the TCP response function should be addressed in the IETF, rather than made as ad hoc decisions by individual implementors or TCP senders. Modifications to the TCP response function would also have implications for transport protocols that use TFRC and other forms of equation-based congestion control, as these congestion control mechanisms directly use the TCP response function [RFC3448].

This proposal for HighSpeed TCP focuses specifically on a proposed change to the TCP response function, and its implications for TCP. This document does not address what we view as a separate fundamental issue, of the mechanisms required to enable best-effort connections to *start* with large initial windows. In our view, while Highspeed TCP proposes a somewhat fundamental change to the TCP response function, at the same time it is a relatively simple change to implement in a single TCP sender, and presents no dangers in terms of congestion collapse. In contrast, in our view, the problem of enabling connections to *start* with large initial windows is inherently more risky and structurally more difficult, requiring some form of explicit feedback from all of the routers along the path. This is another reason why we would propose addressing the problem of starting with large initial windows separately, and on a separate timetable, from the problem of modifying the TCP response function. 


\section{The Problem Description}

This section describes the number of round-trip times between congestion events required for a standard TCP flow to achieve an average throughput of B bps, given packets of D bytes and a roundtrip time of $R$ seconds. A congestion event refers to a window of data with one or more dropped or ECN-marked packets (where ECN stands for Explicit Congestion Notification).

From Appendix A, achieving an average TCP throughput of $B$ bps requires a loss event at most every BR/(12D) round-trip times. This is illustrated in Table 1 , for $R=0.1$ seconds and $D=1500$ bytes. The table also gives the average congestion window $W$ of $B R /(8 D)$, and the steady-state packet drop rate $\mathrm{P}$ of $1.5 / \mathrm{W}^{\wedge} 2$.

$\begin{array}{cccc}\text { TCP Throughput (Mbps) } & \text { RTTS Between Losses } & \text { W } & \text { P } \\ ------- & ---- \\ 1 & 5.5 & 8.3 & 0.02 \\ 10 & 55.5 & 83.3 & 0.0002 \\ 100 & 555.5 & 833.3 & 0.000002 \\ 1000 & 5555.5 & 8333.3 & 0.00000002 \\ 10000 & 55555.5 & 83333.3 & 0.0000000002\end{array}$

Table 1: RTTs Between Congestion Events for Standard TCP, for 1500-Byte Packets and a Round-Trip Time of 0.1 Seconds.

This document proposes Highspeed TCP, a minimal modification to TCP' S increase and decrease parameters, for TCP connections with larger congestion windows, to allow TCP to achieve high throughput with more realistic requirements for the steady-state packet drop rate. Equivalently, Highspeed TCP has more realistic requirements for the number of round-trip times between loss events.

3. Design Guidelines

Our proposal for Highspeed TCP is motivated by the following requirements:

* Achieve high per-connection throughput without requiring unrealistically low packet loss rates.

* Reach high throughput reasonably quickly when in slow-start.

* Reach high throughput without overly long delays when recovering from multiple retransmit timeouts, or when ramping-up from a period with small congestion windows. 
* No additional feedback or support required from routers:

For example, the goal is for acceptable performance in both ECNcapable and non-ECN-Capable environments, and with Drop-Tail as well as with Active Queue Management such as RED in the routers.

* No additional feedback required from TCP receivers.

* TCP-compatible performance in environments with moderate or high congestion (e.g., packet drop rates of $1 \%$ or higher):

Equivalently, the requirement is that there be no additional load on the network (in terms of increased packet drop rates) in environments with moderate or high congestion.

* Performance at least as good as standard TCP in environments with moderate or high congestion.

* Acceptable transient performance, in terms of increases in the congestion window in one round-trip time, responses to severe congestion, and convergence times to fairness.

Currently, users wishing to achieve throughputs of 1 Gbps or more typically open up multiple TCP connections in parallel, or use MulTCP [CO98, GRK99], which behaves roughly like the aggregate of $\mathrm{N}$ virtual TCP connections. While this approach suffices for the occasional user on well-provisioned links, it leaves the parameter $\mathrm{N}$ to be determined by the user, and results in more aggressive performance and higher steady-state packet drop rates if used in environments with periods of moderate or high congestion. We believe that a new approach is needed that offers more flexibility, more effectively scales to a wide range of available bandwidths, and competes more fairly with standard TCP in congested environments.

4. Non-Goals

The following are explicitly *not* goals of our work:

* Non-goal: TCP-compatible performance in environments with very low packet drop rates.

We note that our proposal does not require, or deliver, TCPcompatible performance in environments with very low packet drop rates, e.g., with packet loss rates of $10^{\wedge}-5$ or $10^{\wedge}-6$. As we discuss later in this document, we assume that standard TCP is unable to make effective use of the available bandwidth in environments with loss 
rates of $10^{\wedge}-6$ in any case, so that it is acceptable and appropriate for Highspeed TCP to perform more aggressively than standard TCP in such an environment.

* Non-goal: Ramping-up more quickly than allowed by slow-start.

It is our belief that ramping-up more quickly than allowed by slowstart would necessitate more explicit feedback from routers along the path. The proposal for Highspeed TCP is focused on changes to TCP that could be effectively deployed in the current Internet environment.

* Non-goal: Avoiding oscillations in environments with only one-way, long-lived flows all with the same round-trip times.

While we agree that attention to oscillatory behavior is useful, avoiding oscillations in aggregate throughput has not been our primary consideration, particularly for simplified environments limited to one-way, long-lived flows all with the same, large roundtrip times. Our assessment is that some oscillatory behavior in these extreme environments is an acceptable price to pay for the other benefits of Highspeed TCP.

5. Modifying the TCP Response Function

The TCP response function, $\mathrm{w}=1.2 /$ sqrt $(\mathrm{p})$, gives $\mathrm{TCP}^{\prime} \mathrm{s}$ average congestion window $w$ in MSS-sized segments, as a function of the steady-state packet drop rate $\mathrm{p}$ [FF98]. This TCP response function is a direct consequence of $\mathrm{TCP}^{\prime} \mathrm{s}$ Additive Increase Multiplicative Decrease (AIMD) mechanisms of increasing the congestion window by roughly one segment per round-trip time in the absence of congestion, and halving the congestion window in response to a round-trip time with a congestion event. This response function for standard TCP is reflected in the table below. In this proposal we restrict our attention to TCP performance in environments with packet loss rates of at most $10^{\wedge}-2$, and so we can ignore the more complex response functions that are required to model TCP performance in more congested environments with retransmit timeouts. From Appendix $A$, an average congestion window of $W$ corresponds to an average of $2 / 3 \mathrm{~W}$ round-trip times between loss events for standard TCP (with the congestion window varying from $2 / 3 \mathrm{~W}$ to $4 / 3 \mathrm{~W}$ ). 


$\begin{array}{ccc}\text { Packet Drop Rate } P & \text { Congestion Window } W & \text { RTTs Between Losses } \\ 10^{\wedge}-2 & 12 & 8 \\ 10^{\wedge}-3 & 38 & 25 \\ 10^{\wedge}-4 & 120 & 80 \\ 10^{\wedge}-5 & 379 & 252 \\ 10^{\wedge}-6 & 1200 & 800 \\ 10^{\wedge}-7 & 3795 & 2530 \\ 10^{\wedge}-8 & 12000 & 8000 \\ 10^{\wedge}-9 & 37948 & 25298 \\ 10^{\wedge}-10 & 120000 & 80000\end{array}$

Table 2: TCP Response Function for Standard TCP. The average congestion window $W$ in MSS-sized segments is given as a function of the packet drop rate $\mathrm{P}$.

To specify a modified response function for Highspeed TCP, we use three parameters, Low_Window, High_Window, and High_P. To ensure TCP compatibility, the Highspeed response function uses the same response function as Standard TCP when the current congestion window is at most Low_Window, and uses the Highspeed response function when the current congestion window is greater than Low_Window. In this document we set Low_Window to 38 MSS-sized segments, corresponding to a packet drop rate of $10^{\wedge}-3$ for TCP.

To specify the upper end of the Highspeed response function, we specify the packet drop rate needed in the Highspeed response function to achieve an average congestion window of 83000 segments. This is roughly the window needed to sustain 10 Gbps throughput, for a TCP connection with the default packet size and round-trip time used earlier in this document. For High_Window set to 83000, we specify High_P of $10^{\wedge}-7$; that is, with Highspeed TCP a packet drop rate of $10^{\wedge}-7$ allows the Highspeed TCP connection to achieve an average congestion window of 83000 segments. We believe that this loss rate sets an achievable target for high-speed environments, while still allowing acceptable fairness for the Highspeed response function when competing with standard TCP in environments with packet drop rates of $10^{\wedge}-4$ or $10^{\wedge} 5$.

For simplicity, for the Highspeed response function we maintain the property that the response function gives a straight line on a loglog scale (as does the response function for standard TCP, for low to moderate congestion). This results in the following response function, for values of the average congestion window $W$ greater than Low_Window:

$\mathrm{W}=(\mathrm{p} / \mathrm{LOW} \text { _P })^{\wedge} \mathrm{S}$ Low_Window, 
for Low_P the packet drop rate corresponding to Low_Window, and for S as following constant [FRS02]:

$$
S=(\log \text { High_Window - log Low_Window)/(log High_P - log Low_P) . }
$$

(In this paper, "log $x "$ refers to the log base 10.) For example, for Low_Window set to 38, we have Low_P of 10^-3 (for compatibility with Standard TCP). Thus, for High_Window set to 83000 and High_P set to 10^-7, we get the following response function:

$$
\mathrm{W}=0.12 / \mathrm{p}^{\wedge} 0.835
$$

This Highspeed response function is illustrated in Table 3 below. For Highspeed TCP, the number of round-trip times between losses, $1 /(\mathrm{pW})$, equals $12.7 \mathrm{~W}^{\wedge} 0.2$, for $\mathrm{W}>38$ segments.

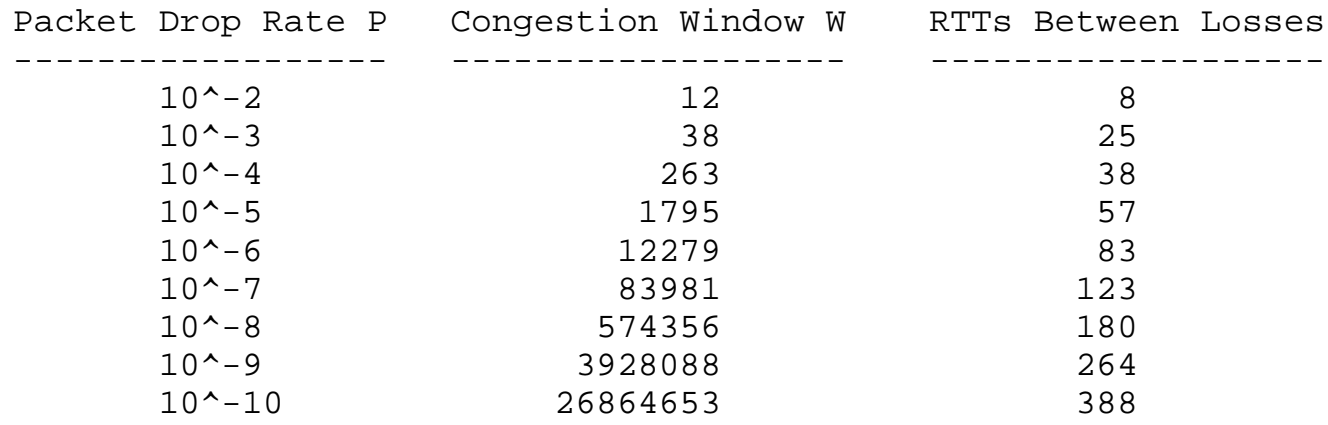

Table 3: TCP Response Function for Highspeed TCP. The average congestion window $W$ in MSS-sized segments is given as a function of the packet drop rate $\mathrm{P}$.

We believe that the problem of backward compatibility with standard TCP requires a response function that is quite close to that of Standard TCP for loss rates of $10^{\wedge}-1,10^{\wedge}-2$, or $10^{\wedge}-3$. We believe, however, that such stringent TCP-compatibility is not required for smaller loss rates, and that an appropriate response function is one that gives a plausible packet drop rate for a connection throughput of 10 Gbps. This also gives a slowly increasing number of round-trip times between loss events as a function of a decreasing packet drop rate.

Another way to look at the Highspeed response function is to consider that Highspeed TCP is roughly emulating the congestion control response of $\mathrm{N}$ parallel $\mathrm{TCP}$ connections, where $\mathrm{N}$ is initially one, and where $\mathrm{N}$ increases as a function of the Highspeed TCP's congestion window. Thus for the Highspeed response function in Equation (1) above, the response function can be viewed as equivalent to that of 
$\mathrm{N}(\mathrm{W})$ parallel TCP connections, where $\mathrm{N}(\mathrm{W})$ varies as a function of the congestion window $W$. Recall that for a single standard TCP connection, the average congestion window equals $1.2 /$ sqrt $(\mathrm{p})$. For $\mathrm{N}$ parallel TCP connections, the aggregate congestion window for the $\mathrm{N}$ connections equals $N^{*} 1.2 / \operatorname{sqrt}(\mathrm{p})$. From the Highspeed response function in Equation (1) and the relationship above, we can derive the following:

$$
N(W)=0.23 * W^{\wedge}(0.4)
$$

for $\mathrm{N}(\mathrm{W})$ the number of parallel TCP connections emulated by the Highspeed TCP response function, and for $N(W)>=1$. This is shown in Table 4 below.

Congestion Window $\mathrm{W}$
1
10
100
1,000
10,000

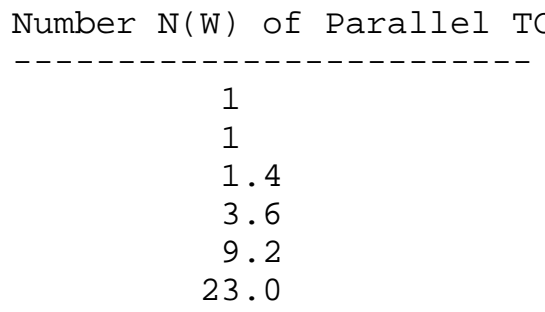

Table 4: Number $\mathrm{N}(\mathrm{W})$ of parallel TCP connections roughly emulated by the Highspeed TCP response function.

In this document, we do not attempt to seriously evaluate the Highspeed response function for congestion windows greater than 100,000 packets. We believe that we will learn more about the requirements for sustaining the throughput of best-effort connections in that range as we gain more experience with Highspeed TCP with congestion windows of thousands and tens of thousands of packets. There also might be limitations to the per-connection throughput that can be realistically achieved for best-effort traffic, in terms of congestion window of hundreds of thousands of packets or more, in the absence of additional support or feedback from the routers along the path.

6. Fairness Implications of the Highspeed Response Function

The Standard and Highspeed Response Functions can be used directly to infer the relative fairness between flows using the two response functions. For example, given a packet drop rate $\mathrm{P}$, assume that Standard TCP has an average congestion window of W_Standard, and Highspeed TCP has a higher average congestion window of W_HighSpeed. 
In this case, a single Highspeed TCP connection is receiving W_HighSpeed/W_Standard times the throughput of a single Standard TCP connection competing in the same environment.

This relative fairness is illustrated below in Table 5, for the parameters used for the Highspeed response function in the section above. The second column gives the relative fairness, for the steady-state packet drop rate specified in the first column. To help calibrate, the third column gives the aggregate average congestion window for the two TCP connections, and the fourth column gives the bandwidth that would be needed by the two connections to achieve that aggregate window and packet drop rate, given $100 \mathrm{~ms}$ round-trip times and 1500-byte packets.

$\begin{array}{cccr}\text { Packet Drop Rate } P & \text { Fairness } & \text { Aggregate Window } & \text { Bandwidth } \\ 10^{\wedge}-2 & 1.0 & 24 & ------- \\ 10^{\wedge}-3 & 1.0 & 76 & 9.1 \mathrm{Mbps} \\ 10^{\wedge}-4 & 2.2 & 383 & 45.9 \mathrm{Mbps} \\ 10^{\wedge}-5 & 4.7 & 2174 & 260.8 \mathrm{Mbps} \\ 10^{\wedge}-6 & 10.2 & 13479 & 1.6 \mathrm{Gbps} \\ 10^{\wedge}-7 & 22.1 & 87776 & 10.5 \mathrm{Gbps}\end{array}$

Table 5: Relative Fairness between the Highspeed and Standard Response Functions.

Thus, for packet drop rates of $10^{\wedge}-4$, a flow with the Highspeed response function can expect to receive 2.2 times the throughput of a flow using the standard response function, given the same round-trip times and packet sizes. With packet drop rates of $10^{\wedge}-6$ (or 10^-7), the unfairness is more severe, and we have entered the regime where a Standard TCP connection requires at most one congestion event every 800 (or 2530) round-trip times in order to make use of the available bandwidth. Our judgement would be that there are not a lot of TCP connections effectively operating in this regime today, with congestion windows of thousands of packets, and that therefore the benefits of the Highspeed response function would outweigh the unfairness that would be experienced by standard TCP in this regime. However, one purpose of this document is to solicit feedback on this issue. The parameter Low_Window determines directly the point of divergence between the Standard and Highspeed Response Functions.

The third column of Table 5, the Aggregate Window, gives the aggregate congestion window of the two competing TCP connections, with Highspeed and Standard TCP, given the packet drop rate specified in the first column. From Table 5, a Highspeed TCP connection would receive ten times the bandwidth of a standard TCP in an environment with a packet drop rate of $10^{\wedge}-6$. This would occur when the two 
flows sharing a single pipe achieved an aggregate window of 13479 packets. Given a round-trip time of $100 \mathrm{~ms}$ and a packet size of 1500 bytes, this would occur with an available bandwidth for the two competing flows of 1.6 Gbps.

Next we consider the time that it takes a standard or Highspeed TCP flow to converge to fairness against a pre-existing Highspeed TCP flow. The worst case for convergence to fairness occurs when a new flow is starting up, competing against a high-bandwidth existing flow, and the new flow suffers a packet drop and exits slow-start while its window is still small. In the worst case, consider that the new flow has entered the congestion avoidance phase while its window is only one packet. A standard TCP flow in congestion avoidance increases its window by at most one packet per round-trip time, and after $\mathrm{N}$ round-trip times has only achieved a window of $\mathrm{N}$ packets (when starting with a window of 1 in the first round-trip time). In contrast, a Highspeed TCP flows increases much faster than a standard TCP flow while in the congestion avoidance phase, and we can expect its convergence to fairness to be much better. This is shown in Table 6 below. The script used to generate this table is given in Appendix $\mathrm{C}$.

$\begin{array}{rrr}\text { RTT } & \text { HS_Window } & \text { Standard_TCP_Window } \\ --- & 131 & 100 \\ 100 & 475 & 200 \\ 200 & 1131 & 300 \\ 300 & 2160 & 400 \\ 400 & 3601 & 500 \\ 500 & 5477 & 600 \\ 600 & 7799 & 700 \\ 700 & 10567 & 800 \\ 800 & 13774 & 900 \\ 900 & 17409 & 1000 \\ 1000 & 21455 & 1100 \\ 1100 & 25893 & 1200 \\ 1200 & 30701 & 1300 \\ 1300 & 35856 & 1400 \\ 1400 & 41336 & 1500 \\ 1500 & 47115 & 1600 \\ 1600 & 53170 & 1700 \\ 1700 & 59477 & 1800 \\ 1800 & 66013 & 1900 \\ 1900 & 72754 & 2000 \\ 2000 & & \end{array}$

Table 6: For a Highspeed and a standard TCP connection, the congestion window during congestion avoidance phase (starting with a congestion window of 1 packet during RTT 1). 
The classic paper on relative fairness is from Chiu and Jain [CJ89]. This paper shows that AIMD (Additive Increase Multiplicative Decrease) converges to fairness in an environment with synchronized congestion events. From [CJ89], it is easy to see that MIMD and AIAD do not converge to fairness in this environment. However, the results of [CJ89] do not apply to an asynchronous environment such as that of the current Internet, where the frequency of congestion feedback can be different for different flows. For example, it has been shown that MIMD converges to fair states in a model with proportional instead of synchronous feedback in terms of packet drops [GV02]. Thus, we are not concerned about abandoning a strict model of AIMD for Highspeed TCP. However, we note that in an environment with Drop-Tail queue management, there is likely to be some synchronization of packet drops. In this environment, the model of completely synchronous feedback does not hold, but the model of completely asynchronous feedback is not accurate either. Fairness in Drop-Tail environments is discussed in more detail in sections 9 and 12 .

7. Translating the Highspeed Response Function into Congestion Control Parameters

For equation-based congestion control such as TFRC, the Highspeed Response Function above could be used directly by the TFRC congestion control mechanism. However, for TCP the Highspeed response function has to be translated into additive increase and multiplicative decrease parameters. The Highspeed response function cannot be achieved by TCP with an additive increase of one segment per roundtrip time and a multiplicative decrease of halving the current congestion window; Highspeed TCP will have to modify either the increase or the decrease parameter, or both. We have concluded that Highspeed TCP is most likely to achieve an acceptable compromise between moderate increases and timely decreases by modifying both the increase and the decrease parameter.

That is, for Highspeed TCP let the congestion window increase by a(w) segments per round-trip time in the absence of congestion, and let the congestion window decrease to $\mathrm{w}(1-\mathrm{b}(\mathrm{w}))$ segments in response to a round-trip time with one or more loss events. Thus, in response to a single acknowledgement Highspeed TCP increases its congestion window in segments as follows:

$\mathrm{w}<-\mathrm{w}+\mathrm{a}(\mathrm{w}) / \mathrm{w}$.

In response to a congestion event, Highspeed TCP decreases as follows:

$\mathrm{w}<-(1-\mathrm{b}(\mathrm{w})) \mathrm{w}$. 
For Standard TCP, $\mathrm{a}(\mathrm{w})=1$ and $\mathrm{b}(\mathrm{w})=1 / 2$, regardless of the value of w. Highspeed TCP uses the same values of $a(w)$ and $b(w)$ for $w<=$ Low_Window. This section specifies $a(w)$ and $b(w)$ for Highspeed TCP for larger values of $w$.

For w = High_Window, we have specified a loss rate of High_P. From [FRS02], or from elementary calculations, this requires the following relationship between $\mathrm{a}(\mathrm{w})$ and $\mathrm{b}(\mathrm{w})$ for $\mathrm{w}=$ High_Window:

$$
\mathrm{a}(\mathrm{w})=\mathrm{High} \_ \text {Window^2 } \text { *High_P } \text { * } 2 \text { * } \mathrm{b}(\mathrm{w}) /(2-\mathrm{b}(\mathrm{w})) \text {. }
$$

We use the parameter High_Decrease to specify the decrease parameter $\mathrm{b}(\mathrm{w})$ for $\mathrm{w}=$ High_Window, and use Equation (2) to derive the increase

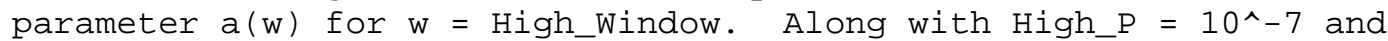
High_Window $=83000$, for example, we specify High_Decrease $=0.1$, specifying that $\mathrm{b}(83000)=0.1$, giving a decrease of $10 \%$ after a congestion event. Equation (2) then gives a (83000) = 72, for an increase of 72 segments, or just under $0.1 \%$, within a round-trip time, for $\mathrm{w}=83000$.

This moderate decrease strikes us as acceptable, particularly when coupled with the role of TCP's ACK-clocking in limiting the sending rate in response to more severe congestion [BBFS01]. A more severe decrease would require a more aggressive increase in the congestion window for a round-trip time without congestion. In particular, a decrease factor High_Decrease of 0.5, as in Standard TCP, would require an increase of 459 segments per round-trip time when $\mathrm{w}=$ 83000 .

Given decrease parameters of $\mathrm{b}(\mathrm{w})=1 / 2$ for $\mathrm{w}=$ Low_Window, and $\mathrm{b}(\mathrm{w})$ = High_Decrease for $w=$ High_Window, we are left to specify the value of $\mathrm{b}(\mathrm{w})$ for other values of $\mathrm{w}>$ Low_Window. From [FRS02], we let $\mathrm{b}(\mathrm{w})$ vary linearly as the log of $\mathrm{w}$, as follows:

$\mathrm{b}(\mathrm{w})=\left(\mathrm{High \_ Decrease}-0.5\right)(\log (\mathrm{W})-\log (\mathrm{W})) /\left(\log \left(\mathrm{W} \_1\right)-\log (\mathrm{W})\right)+$ 0.5 ,

for $W$ = Low_window and $W_{-1}=$ High_window. The increase parameter a $(w)$ can then be computed as follows:

$\mathrm{a}(\mathrm{w})=\mathrm{w}^{\wedge} 2 \star \mathrm{p}(\mathrm{w}) \star 2 \star \mathrm{b}(\mathrm{w}) /(2-\mathrm{b}(\mathrm{w}))$,

for $\mathrm{p}(\mathrm{w})$ the packet drop rate for congestion window w. From inverting Equation (1), we get $p(w)$ as follows:

$\mathrm{p}(\mathrm{w})=0.078 / \mathrm{w}^{\wedge} 1.2$ 
We assume that experimental implementations of Highspeed TCP for further investigation will use a pre-computed look-up table for finding $a(w)$ and $b(w)$. For example, the implementation from Tom Dunigan adjusts the $\mathrm{a}(\mathrm{w})$ and $\mathrm{b}(\mathrm{w})$ parameters every 0.1 seconds. In the appendix we give such a table for our default values of Low_Window $=38$, High_Window $=83,000$, High_P $=10^{\wedge}-7$, and High_Decrease $=0.1$. These are also the default values in the NS simulator; example simulations in NS can be run with the command "./test-all-tcpHighspeed" in the directory tcl/test.

8. An alternate, linear response functions

In this section we explore an alternate, linear response function for Highspeed TCP that has been proposed by a number of other people, in particular by Glenn Vinnicombe and Tom Kelly. Similarly, it has been suggested by others that a less "ad-hoc" guideline for a response function for Highspeed TCP would be to specify a constant value for the number of round-trip times between congestion events.

Assume that we keep the value of Low_Window as 38 MSS-sized segments, indicating when the Highspeed response function diverges from the current TCP response function, but that we modify the High_Window and High_P parameters that specify the upper range of the Highspeed response function. In particular, consider the response function given by High_Window $=380,000$ and High_P $=10^{\wedge}-7$, with Low_Window = 38 and Low_P $=10^{\wedge}-3$ as before.

Using the equations in Section 5, this would give the following Linear response function, for $w>$ Low_Window:

$\mathrm{W}=0.038 / \mathrm{p}$

This Linear Highspeed response function is illustrated in Table 7 below. For Highspeed TCP, the number of round-trip times between losses, 1/(pW), equals $1 / 0.38$, or equivalently, 26 , for $W>38$ segments. 


$\begin{array}{ccc}\text { Packet Drop Rate } P & \text { Congestion Window W } & \text { RTTs Between Losses } \\ 10^{\wedge}-2 & 12 & 8 \\ 10^{\wedge}-3 & 38 & 26 \\ 10^{\wedge}-4 & 380 & 26 \\ 10^{\wedge}-5 & 3800 & 26 \\ 10^{\wedge}-6 & 38000 & 26 \\ 10^{\wedge}-7 & 380000 & 26 \\ 10^{\wedge}-8 & 3800000 & 26 \\ 10^{\wedge}-9 & 38000000 & 26 \\ 10^{\wedge}-10 & 380000000 & 26\end{array}$

Table 7: An Alternate, Linear TCP Response Function for HighSpeed TCP. The average congestion window $W$ in MSS-sized segments is given as a function of the packet drop rate $\mathrm{P}$.

Given a constant decrease $b(w)$ of $1 / 2$, this would give an increase a (w) of w/Low_Window, or equivalently, a constant increase of 1/Low_Window packets per acknowledgement, for $w>$ Low_Window. Another possibility is Scalable TCP [K03], which uses a fixed decrease $b(w)$ of $1 / 8$ and a fixed increase per acknowledgement of 0.01 . This gives an increase a(w) per window of $0.005 \mathrm{w}$, for a TCP with delayed acknowledgements, for pure MIMD.

The relative fairness between the alternate Linear response function and the standard TCP response function is illustrated below in Table 8 .

$\begin{array}{cccr}\text { Packet Drop Rate } P & \text { Fairness } & \text { Aggregate Window } & \text { Bandwidth } \\ \text { 10^-2 } & 1.0 & 24 & ------- \\ 10^{\wedge}-3 & 1.0 & 76 & 9.8 \mathrm{Mbps} \\ 10^{\wedge}-4 & 3.2 & 500 & 60.0 \mathrm{Mbps} \\ 10^{\wedge}-5 & 15.1 & 4179 & 501.4 \mathrm{Mbps} \\ 10^{\wedge}-6 & 31.6 & 39200 & 4.7 \mathrm{Gbps} \\ 10^{\wedge}-7 & 100.1 & 383795 & 46.0 \mathrm{Gbps}\end{array}$

Table 8: Relative Fairness between the Linear Highspeed and Standard Response Functions.

One attraction of the linear response function is that it is scaleinvariant, with a fixed increase in the congestion window per acknowledgement, and a fixed number of round-trip times between loss events. My own assumption would be that having a fixed length for the congestion epoch in round-trip times, regardless of the packet drop rate, would be a poor fit for an imprecise and imperfect world with routers with a range of queue management mechanisms, such as the Drop-Tail queue management that is common today. For example, a 
response function with a fixed length for the congestion epoch in round-trip times might give less clearly-differentiated feedback in an environment with steady-state background losses at fixed intervals for all flows (as might occur with a wireless link with occasional short error bursts, giving losses for all flows every $\mathrm{N}$ seconds regardless of their sending rate).

While it is not a goal to have perfect fairness in an environment with synchronized losses, it would be good to have moderately acceptable performance in this regime. This goal might argue against a response function with a constant number of round-trip times between congestion events. However, this is a question that could clearly use additional research and investigation. In addition, flows with different round-trip times would have different time durations for congestion epochs even in the model with a linear response function.

The third column of Table 8, the Aggregate Window, gives the aggregate congestion window of two competing TCP connections, one with Linear Highspeed TCP and one with Standard TCP, given the packet drop rate specified in the first column. From Table 8, a Linear Highspeed TCP connection would receive fifteen times the bandwidth of a Standard TCP in an environment with a packet drop rate of $10^{\wedge}-5$. This would occur when the two flows sharing a single pipe achieved an aggregate window of 4179 packets. Given a round-trip time of $100 \mathrm{~ms}$ and a packet size of 1500 bytes, this would occur with an available bandwidth for the two competing flows of $501 \mathrm{Mbps}$. Thus, because the Linear Highspeed TCP is more aggressive than the Highspeed TCP proposed above, it also is less fair when competing with standard TCP in a high-bandwidth environment.

9. Tradeoffs for Choosing Congestion Control Parameters

A range of metrics can be used for evaluating choices for congestion control parameters for Highspeed TCP. My assumption in this section is that for a response function of the form $w=c / p^{\wedge} d$, for constant $c$ and exponent $d$, the only response functions that would be considered are response functions with $1 / 2<=d<=1$. The two ends of this spectrum are represented by current TCP, with $d=1 / 2$, and by the linear response function described in section 8 above, with $d=1$. Highspeed TCP lies somewhere in the middle of the spectrum, with $d=$ 0.835 .

Response functions with exponents less than 1/2 can be eliminated from consideration because they would be even worse than standard TCP in accommodating connections with high congestion windows. 
9.1. The Number of Round-Trip Times between Loss Events

Response functions with exponents greater than 1 can be eliminated from consideration because for these response functions, the number of round-trip times between loss events decreases as congestion decreases. For a response function of $\mathrm{w}=\mathrm{c} / \mathrm{p}^{\wedge} \mathrm{d}$, with one loss event or congestion event every $1 / \mathrm{p}$ packets, the number of round-trip times between loss events is $\mathrm{w}^{\wedge}((1 / \mathrm{d})-1) / \mathrm{C}^{\wedge}(1 / \mathrm{d})$. Thus, for standard $\mathrm{TCP}$ the number of round-trip times between loss events is linear in $w$. In contrast, one attraction of the linear response function, as described in section 8 above, is that it is scale-invariant, in terms of a fixed increase in the congestion window per acknowledgement, and a fixed number of round-trip times between loss events.

However, for a response function with $\mathrm{d}>1$, the number of roundtrip times between loss events would be proportional to $\mathrm{w}^{\wedge}((1 / \mathrm{d})-1)$, for a negative exponent $((1 / d)-1)$, setting smaller as w increases. This would seem undesirable.

9.2. The Number of Packet Drops per Loss Event, with Drop-Tail

A TCP connection increases its sending rate by a(w) packets per round-trip time, and in a Drop-Tail environment, this is likely to result in a(w) dropped packets during a single loss event. One attraction of standard TCP is that it has a fixed increase per round-trip time of one packet, minimizing the number of packets that would be dropped in a Drop-Tail environment. For an environment with some form of Active Queue Management, and in particular for an environment that uses ECN, the number of packets dropped in a single congestion event would not be a problem. However, even in these environments, larger increases in the sending rate per round-trip time result in larger stresses on the ability of the queues in the router to absorb the fluctuations.

Highspeed TCP plays a middle ground between the metrics of a moderate number of round-trip times between loss events, and a moderate increase in the sending rate per round-trip time. As shown in Appendix B, for a congestion window of 83,000 packets, Highspeed TCP increases its sending rate by 70 packets per round-trip time, resulting in at most 70 packet drops when the buffer overflows in a Drop-Tail environment. This increased aggressiveness is the price paid by Highspeed TCP for its increased scalability. A large number of packets dropped per congestion event could result in synchronized drops from multiple flows, with a possible loss of throughput as a result. 
Scalable TCP has an increase a(w) of $0.005 \mathrm{w}$ packets per round-trip time. For a congestion window of 83,000 packets, this gives an increase of 415 packets per round-trip time, resulting in roughly 415 packet drops per congestion event in a Drop-Tail environment.

Thus, Highspeed TCP and its variants place increased demands on queue management in routers, relative to standard TCP. (This is rather similar to the increased demands on queue management that would result from using $\mathrm{N}$ parallel $\mathrm{TCP}$ connections instead of a single Standard TCP connection.)

10. Related Issues

10.1. Slow-Start

A companion internet-draft on "Limited Slow-Start for TCP with Large Congestion Windows" [F02b] proposes a modification to TCP's slowstart procedure that can significantly improve the performance of TCP connections slow-starting up to large congestion windows. For TCP connections that are able to use congestion windows of thousands (or tens of thousands) of MSS-sized segments (for MSS the sender's MAXIMUM SEGMENT SIZE), the current slow-start procedure can result in increasing the congestion window by thousands of segments in a single round-trip time. Such an increase can easily result in thousands of packets being dropped in one round-trip time. This is often counter-productive for the TCP flow itself, and is also hard on the rest of the traffic sharing the congested link.

[F02b] proposes Limited Slow-Start, limiting the number of segments by which the congestion window is increased for one window of data during slow-start, in order to improve performance for TCP connections with large congestion windows. We have separated out Limited Slow-Start to a separate draft because it can be used both with Standard or with Highspeed TCP.

Limited Slow-Start is illustrated in the NS simulator, for snapshots after May 1, 2002, in the tests "./test-all-tcpHighspeed tcp1A" and "./test-all-tcpHighspeed tcpHighspeedl" in the subdirectory "tcl/lib".

In order for best-effort flows to safely start-up faster than slowstart, e.g., in future high-bandwidth networks, we believe that it would be necessary for the flow to have explicit feedback from the routers along the path. There are a number of proposals for this, ranging from a minimal proposal for an IP option that allows TCP SYN packets to collect information from routers along the path about the allowed initial sending rate [J02], to proposals with more power that require more fine-tuned and continuous feedback from routers. These 
proposals are all somewhat longer-term proposals than the Highspeed TCP proposal in this document, requiring longer lead times and more coordination for deployment, and will be discussed in later documents.

\subsection{Limiting burstiness on short time scales}

Because the congestion window achieved by a Highspeed TCP connection could be quite large, there is a possibility for the sender to send a large burst of packets in response to a single acknowledgement. This could happen, for example, when there is congestion or reordering on the reverse path, and the sender receives an acknowledgement acknowledging hundreds or thousands of new packets. Such a burst would also result if the application was idle for a short period of time less than a round-trip time, and then suddenly had lots of data available to send. In this case, it would be useful for the Highspeed TCP connection to have some method for limiting bursts.

In this document, we do not specify TCP mechanisms for reducing the short-term burstiness. One possible mechanism is to use some form of rate-based pacing, and another possibility is to use maxburst, which limits the number of packets that are sent in response to a single acknowledgement. We would caution, however, against a permanent reduction in the congestion window as a mechanism for limiting short-term bursts. Such a mechanism has been deployed in some TCP stacks, and our view would be that using permanent reductions of the congestion window to reduce transient bursts would be a bad idea [Fl03].

\subsection{Other limitations on window size}

The TCP header uses a 16-bit field to report the receive window size to the sender. Unmodified, this allows a window size of at most $2 * \star 16=65 \mathrm{~K}$ bytes. With window scaling, the maximum window size is $2 * \star 30=1073 \mathrm{M}$ bytes [RFC 1323]. Given 1500-byte packets, this allows a window of up to 715,000 packets.

10.4. Implementation issues

One implementation issue that has been raised with Highspeed $\mathrm{TCP}$ is that with congestion windows of $4 \mathrm{MB}$ or more, the handling of successive SACK packets after a packet is dropped becomes very timeconsuming at the TCP sender [S03]. Tom Kelly's Scalable TCP includes a "SACK Fast Path" patch that addresses this problem.

The issues addressed in the Web100 project, the Net100 project, and related projects about the tuning necessary to achieve high bandwidth data rates with TCP apply to Highspeed TCP as well [Net100, Web100]. 


\section{Deployment issues}

\subsection{Deployment issues of Highspeed TCP}

We do not claim that the Highspeed TCP modification to TCP described in this paper is an optimal transport protocol for high-bandwidth environments. Based on our experiences with Highspeed TCP in the NS simulator [NS], on simulation studies [SA03], and on experimental reports [ABLLS03,D02, CC03,F03], we believe that Highspeed TCP improves the performance of TCP in high-bandwidth environments, and we are documenting it for the benefit of the IETF community. We encourage the use of Highspeed TCP, and of its underlying response function, and we further encourage feedback about operational experiences with this or related modifications.

We note that in environments typical of much of the current Internet, Highspeed TCP behaves exactly as does Standard TCP today. This is the case any time the congestion window is less than 38 segments.

$\begin{array}{rccc}\text { Bandwidth } & \text { Avg Cwnd } \mathrm{w} \text { (pkts) } & \text { Increase a (w) } & \text { Decrease b }(\mathrm{w}) \\ ------- & ------------- & --------- & ---------- \\ 1.5 \text { Mbps } & 12.5 & 1 & 0.50 \\ 10 \mathrm{Mbps} & 83 & 1 & 0.50 \\ 100 \mathrm{Mbps} & 833 & 6 & 0.35 \\ 1 \text { Gbps } & 8333 & 26 & 0.22 \\ 10 \mathrm{Gbps} & 83333 & 70 & 0.10\end{array}$

Table 9: Performance of a Highspeed TCP connection

To help calibrate, Table 9 considers a TCP connection with 1500-byte packets, an RTT of $100 \mathrm{~ms}$ (including average queueing delay), and no competing traffic, and shows the average congestion window if that TCP connection had a pipe all to itself and fully used the link bandwidth, for a range of bandwidths for the pipe. This assumes that the TCP connection would use Table 12 in determining its increase and decrease parameters. The first column of Table 9 gives the bandwidth, and the second column gives the average congestion window w needed to utilize that bandwidth. The third column shows the increase $a(w)$ in segments per RTT for window $w$. The fourth column shows the decrease $\mathrm{b}(\mathrm{w})$ for that window $\mathrm{w}$ (where the TCP sender decreases the congestion window from $w$ to $w(1-b(w))$ segments after a loss event). When a loss occurs we note that the actual congestion window is likely to be greater than the average congestion window w in column 2, so the decrease parameter used could be slightly smaller than the one given in column 4 of Table 9.

Table 9 shows that a Highspeed TCP over a 10 Mbps link behaves exactly the same as a standard TCP connection, even in the absence of 
competing traffic. One can think of the congestion window staying generally in the range of 55 to 110 segments, with the Highspeed TCP behavior being exactly the same as the behavior of standard TCP. (If the congestion window is ever 128 segments or more, then the Highspeed TCP increases by two segments per RTT instead of by one, and uses a decrease parameter of 0.44 instead of 0.50.$)$

Table 9 shows that for a Highspeed TCP connection over a 100 Mbps link, with no competing traffic, Highspeed TCP behaves roughly as aggressively as six parallel TCP connections, increasing its congestion window by roughly six segments per round-trip time, and with a decrease parameter of roughly $1 / 3$ (corresponding to decreasing down to 2/3-rds of its old congestion window, rather than to half, in response to a loss event).

For a standard TCP connection in this environment, the congestion window could be thought of as generally varying in the range of 550 to 1100 segments, with an average packet drop rate of $2.2 * 10^{\wedge}-6$ (corresponding to a bit error rate of $1.8 * 10^{\wedge}-10$ ), or equivalently, roughly 55 seconds between congestion events. While a standard TCP connection could sustain such a low packet drop rate in a carefully controlled environment with minimal competing traffic, we would contend that in an uncontrolled best-effort environment with even a small amount of competing traffic, the occasional congestion events from smaller competing flows could easily be sufficient to prevent a Standard TCP flow with no lower-speed bottlenecks from fully utilizing the available bandwidth of the underutilized $100 \mathrm{Mbps}$ link.

That is, we would contend that in the environment of $100 \mathrm{Mbps}$ links with a significant amount of available bandwidth, standard TCP would sometimes be unable to fully utilize the link bandwidth, and that Highspeed TCP would be an improvement in this regard. We would further contend that in this environment, the behavior of Highspeed TCP is sufficiently close to that of Standard TCP that Highspeed TCP would be safe to deploy in the current Internet. We note that Highspeed TCP can only use high congestion windows if allowed by the receiver's advertised window size. As a result, even if Highspeed TCP was ubiquitously deployed in the Internet, the impact would be limited to those TCP connections with an advertised window from the receiver of 118 MSS or larger.

We do not believe that the deployment of Highspeed TCP would serve as a block to the possible deployment of alternate experimental protocols for high-speed congestion control, such as Scalable TCP, $\mathrm{XCP}$ [KHR02], or FAST TCP [JWL03]. In particular, we don't expect Highspeed TCP to interact any more poorly with alternative experimental proposals than would the $\mathrm{N}$ parallel TCP connections commonly used today in the absence of Highspeed TCP. 


\subsection{Deployment issues of Scalable TCP}

We believe that Scalable TCP and Highspeed TCP have sufficiently similar response functions that they could easily coexist in the Internet. However, we have not investigated Scalable TCP sufficiently to be able to claim, in this document, that Scalable TCP is safe for a widespread deployment in the current Internet.

$\begin{array}{rccc}\text { Bandwidth } & \text { Avg Cwnd w (pkts) } & \text { Increase a (w) } & \text { Decrease b (w) } \\ -------- & ------------- & ---------- & ---------- \\ 1.5 \text { Mbps } & 12.5 & 1 & 0.50 \\ 10 \mathrm{Mbps} & 83 & 0.4 & 0.125 \\ 100 \mathrm{Mbps} & 833 & 4.1 & 0.125 \\ 1 \mathrm{Gbps} & 8333 & 41.6 & 0.125 \\ 10 \mathrm{Gbps} & 83333 & 416.5 & 0.125\end{array}$

Table 10: Performance of a Scalable TCP connection.

Table 10 shows the performance of a Scalable TCP connection with 1500-byte packets, an RTT of $100 \mathrm{~ms}$ (including average queueing delay), and no competing traffic. The TCP connection is assumed to use delayed acknowledgements. The first column of Table 10 gives the bandwidth, the second column gives the average congestion window needed to utilize that bandwidth, and the third and fourth columns give the increase and decrease parameters.

Note that even in an environment with a 10 Mbps link, Scalable TCP's behavior is considerably different from that of standard TCP. The increase parameter is smaller than that of Standard TCP, and the decrease is smaller also, 1/8-th instead of $1 / 2$. That is, for 10 Mbps links, Scalable TCP increases less aggressively than Standard TCP or Highspeed TCP, but decreases less aggressively as well.

In an environment with a 100 Mbps link, Scalable TCP has an increase parameter of roughly four segments per round-trip time, with the same decrease parameter of 1/8-th. A comparison of Tables 9 and 10 shows that for this scenario of $100 \mathrm{Mbps}$ links, Highspeed TCP increases more aggressively than scalable TCP.

Next we consider the relative fairness between standard TCP, Highspeed TCP and Scalable TCP. The relative fairness between Highspeed TCP and Standard TCP was shown in Table 5 earlier in this document, and the relative fairness between scalable TCP and standard TCP was shown in Table 8. Following the approach in section 6, for a given packet drop rate $\mathrm{p}$, for $\mathrm{p}<10^{\wedge}-3$, we can estimate the relative fairness between Scalable and Highspeed TCP as

W_Scalable/W_Highspeed. This relative fairness is shown in Table 11 below. The bandwidth in the last column of Table 11 is the aggregate 
bandwidth of the two competing flows given $100 \mathrm{~ms}$ round-trip times and 1500-byte packets.

$\begin{array}{cccr}\text { Packet Drop Rate P } & \text { Fairness } & \text { Aggregate Window } & \text { Bandwidth } \\ -10^{\wedge}-2 & 1.0 & 24 & -8.8 \mathrm{Mbps} \\ 10^{\wedge}-3 & 1.0 & 76 & 9.1 \mathrm{Mbps} \\ 10^{\wedge}-4 & 1.4 & 643 & 77.1 \mathrm{Mbps} \\ 10^{\wedge}-5 & 2.1 & 5595 & 671.4 \mathrm{Mbps} \\ 10^{\wedge}-6 & 3.1 & 50279 & 6.0 \mathrm{Gbps} \\ 10^{\wedge}-7 & 4.5 & 463981 & 55.7 \mathrm{Gbps}\end{array}$

Table 11: Relative Fairness between the Scalable and Highspeed Response Functions.

The second row of Table 11 shows that for a Scalable TCP and a Highspeed TCP flow competing in an environment with $100 \mathrm{~ms}$ RTS and a $10 \mathrm{Mbps}$ pipe, the two flows would receive essentially the same bandwidth. The next row shows that for a scalable TCP and a Highspeed TCP flow competing in an environment with $100 \mathrm{~ms}$ RTTs and a 100 Mbps pipe, the scalable TCP flow would receive roughly 50\% more bandwidth than would Highspeed TCP. Table 11 shows the relative fairness in higher-bandwidth environments as well. This relative fairness seems sufficient that there should be no problems with Scalable TCP and Highspeed TCP coexisting in the same environment as Experimental variants of TCP.

We note that one question that requires more investigation with Scalable TCP is that of convergence to fairness in environments with Drop-Tail queue management.

12. Related Work in HighSpeed TCP

Highspeed TCP has been separately investigated in simulations by Sylvia Ratnasamy and by Evandro de Souza [SA03]. The simulations in [SA03] verify the fairness properties of Highspeed TCP when sharing a link with standard TCP.

These simulations explore the relative fairness of Highspeed TCP flows when competing with standard TCP. The simulation environment includes background forward and reverse-path TCP traffic limited by the TCP receive window, along with a small amount of forward and reverse-path traffic from the web traffic generator. Most of the simulations so far explore performance on a simple dumbbell topology with a 1 Gbps link with a propagation delay of $50 \mathrm{~ms}$. Simulations have been run with Adaptive RED and with DropTail queue management. 
The simulations in [SA03] explore performance with a varying number of competing flows, with the competing traffic being all standard TCP; all Highspeed TCP; or a mix of standard and Highspeed TCP. For the simulations in [SA03] with RED queue management, the relative fairness between standard and Highspeed TCP is consistent with the relative fairness predicted in Table 5. For the simulations with Drop Tail queues, the relative fairness is more skewed, with the Highspeed TCP flows receiving an even larger share of the link bandwidth. This is not surprising; with Active Queue Management at the congested link, the fraction of packet drops received by each flow should be roughly proportional to that flow's share of the link bandwidth, while this property no longer holds with Drop Tail queue management. We also note that relative fairness in simulations with Drop Tail queue management can sometimes depend on small details of the simulation scenario, and that Drop Tail simulations need special care to avoid phase effects [F92].

[SA03] explores the bandwidth 'stolen' by Highspeed TCP from standard TCP by exploring the fraction of the link bandwidth $\mathrm{N}$ standard TCP flows receive when competing against $\mathrm{N}$ other standard TCP flows, and comparing this to the fraction of the link bandwidth the $\mathrm{N}$ standard TCP flows receive when competing against $\mathrm{N}$ Highspeed TCP flows. For the 1 Gbps simulation scenarios dominated by long-lived traffic, a small number of standard TCP flows are able to achieve high link utilization, and the Highspeed TCP flows can be viewed as stealing bandwidth from the competing standard TCP flows, as predicted in Section 6 on the Fairness Implications of the Highspeed Response Function. However, [SA03] shows that when even a small fraction of the link bandwidth is used by more bursty, short TCP connections, the standard TCP flows are unable to achieve high link utilization, and the Highspeed TCP flows in this case are not 'stealing' bandwidth from the standard TCP flows, but instead are using bandwidth that otherwise would not be utilized.

The conclusions of [SA03] are that "Highspeed TCP behaved as forseen by its response function, and appears to be a real and viable option for use on high-speed wide area TCP connections."

Future work that could be explored in more detail includes convergence times after new flows start-up; recovery time after a transient outage; the response to sudden severe congestion, and investigations of the potential for oscillations. We invite contributions from others in this work. 


\section{Relationship to other Work}

Our assumption is that Highspeed TCP will be used with the TCP SACK option, and also with the increased Initial Window of three or four segments, as allowed by [RFC3390]. For paths that have substantial reordering, TCP performance would be greatly improved by some of the mechanisms still in the research stages for robust performance in the presence of reordered packets.

Our view is that Highspeed TCP is largely orthogonal to proposals for higher PMTU (Path MTU) values [M02]. Unlike changes to the PMTU, Highspeed TCP does not require any changes in the network or at the TCP receiver, and works well in the current Internet. Our assumption is that Highspeed TCP would be useful even with larger values for the PMTU. Unlike the current congestion window, the PMTU gives no information about the bandwidth-delay product available to that particular flow.

A related approach is that of a virtual MTU, where the actual MTU of the path might be limited [VMSS,S02]. The virtual MTU approach has not been fully investigated, and we do not explore the virtual MTU approach further in this document.

\section{Conclusions}

This document has proposed Highspeed TCP, a modification to TCP' S congestion control mechanism for use with TCP connections with large congestion windows. We have explored this proposal in simulations, and others have explored Highspeed TCP with experiments, and we believe Highspeed TCP to be safe to deploy on the current Internet. We would welcome additional analysis, simulations, and particularly, experimentation. More information on simulations and experiments is available from the Highspeed TCP Web Page [HSTCP]. There are several independent implementations of Highspeed TCP [D02,F03] and of Scalable TCP [K03] for further investigation.

\section{Acknowledgements}

The Highspeed TCP proposal is from joint work with Sylvia Ratnasamy and scott Shenker (and was initiated by scott Shenker). Additional investigations of Highspeed TCP were joint work with Evandro de Souza and Deb Agarwal. We thank Tom Dunigan for the implementation in the Linux 2.4.16 Web100 kernel, and for resulting experimentation with Highspeed TCP. We are grateful to the End-to-End Research Group, the members of the Transport Area Working Group, and to members of the IPAM program in Large Scale Communication Networks for feedback. We thank Glenn Vinnicombe for framing the Linear response function in the parameters of Highspeed TCP. We are also grateful for 
contributions and feedback from the following individuals: Les Cottrell, Mitchell Erblich, Jeffrey Hsu, Tom Kelly, Chuck Jackson, Matt Mathis, Jitendra Padhye, Andrew Reiter, Stanislav Shalunov, Alex Solan, Paul Sutter, Brian Tierney, Joe Touch.

16. Normative References

[RFC2581] Allman, M., Paxson, V. and W. Stevens, "TCP Congestion Control", RFC 2581, April 1999.

17. Informative References

[ABLLS03] A. Antony, J. Blom, C. de Laat, J. Lee, and W. Sjouw, "Microscopic Examination of TCP Flows over Transatlantic Links", iGrid2002 special issue, Future Generation Computer systems, volume 19 issue 6 (2003), URL

"http://www.science.uva.nl/ delaat/techrep-2003-2tcp.pdf".

[BBFS01] Deepak Bansal, Hari Balakrishnan, Sally Floyd, and Scott Shenker, "Dynamic Behavior of Slowly-Responsive Congestion Control Algorithms", SIGCOMM 2001, August 2001.

[CC03] Fabrizio Coccetti and Les Cottrell, "TCP Stack Measurements on Lightly Loaded Testbeds", 2003. URL "http://www-iepm.slac.stanford.edu/monitoring/bulk/fast/" .

[CJ89] D. Chiu and R. Jain, "Analysis of the Increase and Decrease Algorithms for Congestion Avoidance in Computer Networks", Computer Networks and ISDN Systems, Vol. 17, pp. 1-14, 1989 .

[C098] J. Crowcroft and P. Oechslin, "Differentiated End-to-end Services using a Weighted Proportional Fair Share TCP", Computer Communication Review, 28(3):53--69, 1998.

[D02] Tom Dunigan, "Floyd's TCP slow-start and AIMD mods", URL "http://www.csm.ornl.gov/ dunigan/net100/floyd.html".

[F03] Gareth Fairey, "High-Speed TCP", 2003. URL "http://www.hep.man.ac.uk/u/garethf/hstcp/".

[F92] S. Floyd and V. Jacobson, "On Traffic Phase Effects in Packet-Switched Gateways, Internetworking: Research and Experience", V.3 N.3, September 1992, p.115-156. URL "http://www.icir.org/floyd/papers.html". 
[Fl03] Sally Floyd, "Re: [Tsvwg] taking NewReno (RFC 2582) to Proposed Standard", Email to the tsvwg mailing list, May $14,2003$.

URLs "http://www1.ietf.org/mail-archive/workinggroups/tsvwg/current/msg04086.html" and

"http://www1.ietf.org/mail-archive/workinggroups/tsvwg/current/msg04087.html".

[FF98] Floyd, S., and Fall, K., "Promoting the Use of End-to-End Congestion Control in the Internet", IEEE/ACM Transactions on Networking, August 1999.

[FRS02] Sally Floyd, Sylvia Ratnasamy, and Scott Shenker, "Modifying TCP's Congestion Control for High Speeds", May 2002. URL "http://wWw.icir.org/floyd/notes.html".

[GRK99] Panos Gevros, Fulvio Risso and Peter Kirstein, "Analysis of a Method for Differential TCP Service". In Proceedings of the IEEE GLOBECOM' 99, Symposium on Global Internet, December 1999, Rio de Janeiro, Brazil.

[GV02] S. Gorinsky and H. Vin, "Extended Analysis of Binary Adjustment Algorithms", Technical Report TR2002-39, Department of Computer Sciences, The University of Texas at Austin, August 2002. URL

"http://www.cs.utexas.edu/users/gorinsky/pubs.html" .

[HSTCP] Highspeed TCP Web Page, URL "http://www.icir.org/floyd/hstcp.html".

[J02] Amit Jain and Sally Floyd, "Quick-Start for TCP and IP", Work in Progress, 2002 .

[JWL03] Cheng Jin, David X. Wei and Steven H. Low, "FAST TCP for High-speed Long-distance Networks", Work in Progress, June 2003 .

[K03] Tom Kelly, "Scalable TCP: Improving Performance in Highspeed Wide Area Networks", February 2003. URL "http://www-lce.eng.cam.ac.uk/ ctk21/scalable/" .

[KHR02] Dina Katabi, Mark Handley, and Charlie Rohrs, "Congestion Control for High Bandwidth-Delay Product Networks", SIGCOMM 2002 .

[M02] Matt Mathis, "Raising the Internet MTU", Web Page, URL "http://www.psc.edu/〜mathis/MTU/" . 
[Net100] The DOE/MICS Net100 project. URL "http://www.csm.ornl.gov/ dunigan/net100/".

[NS] The NS Simulator, "http://www.isi.edu/nsnam/ns/".

[RFC 1323] Jacobson, V., Braden, R. and D. Borman, "TCP Extensions for High Performance", RFC 1323, May 1992.

[RFC3390] Allman, M., Floyd, S. and C., Partridge, "Increasing TCP'S Initial Window", $\operatorname{RFC} 3390$, October 2002.

[RFC3448] Handley, M., Padhye, J., Floyd, S. and J. Widmer, "TCP Friendly Rate Control (TFRC): Protocol specification", RFC 3448, January 2003 .

[SA03] Souza, E. and D.A., Agarwal, "A HighSpeed TCP Study: Characteristics and Deployment Issues", LBNL Technical Report LBNL-53215. URL "http://www.icir.org/floyd/hstcp.html".

[S02] Stanislav Shalunov, "TCP Armonk", Work in Progress, 2002, URL "http://www.internet2.edu/ shalunov/tcpar/".

[S03] Alex Solan, private communication, 2003.

[VMSS] "Web100 at ORNL", Web Page, "http://www.csm.ornl.gov/ dunigan/netperf/web100.html".

[Web100] The Web100 project. URL "http://www.web100.org/".

18. Security Considerations

This proposal makes no changes to the underlying security of TCP.

19. IANA Considerations

There are no IANA considerations regarding this document. 
A. TCP's Loss Event Rate in steady-State

This section gives the number of round-trip times between congestion events for a TCP flow with D-byte packets, for $D=1500$, as a function of the connection's average throughput B in bps. To achieve this average throughput $B$, a TCP connection with round-trip time $R$ in seconds requires an average congestion window $w$ of $B R /(8 D)$ segments.

In steady-state, $\mathrm{TCP}^{\prime} \mathrm{s}$ average congestion window $\mathrm{w}$ is roughly 1.2/sqrt $(p)$ segments. This is equivalent to a lost event at most once every $1 / \mathrm{p}$ packets, or at most once every $1 /(\mathrm{pw})=\mathrm{w} / 1.5 \mathrm{round}-$ trip times. Substituting for $w$, this is a loss event at most every $(\mathrm{BR}) / 12 \mathrm{D})$ round-trip times.

An an example, for $\mathrm{R}=0.1$ seconds and $\mathrm{D}=1500$ bytes, this gives B/180000 round-trip times between loss events. 
B. A table for $a(w)$ and $b(w)$.

This section gives a table for the increase and decrease parameters $\mathrm{a}(\mathrm{w})$ and $\mathrm{b}(\mathrm{w})$ for Highspeed TCP, for the default values of Low_Window $=38$, High_Window $=83000$, High_P $=10^{\wedge}-7$, and High_Decrease $=0.1$.

$\begin{array}{rrr}\text { w } & \text { a (w) } & \text { b (w) } \\ ---- & ---- & ---- \\ 38 & 1 & 0.50 \\ 118 & 2 & 0.44 \\ 221 & 3 & 0.41 \\ 347 & 4 & 0.38 \\ 495 & 5 & 0.37 \\ 663 & 6 & 0.35 \\ 851 & 7 & 0.34 \\ 1058 & 8 & 0.33 \\ 1284 & 9 & 0.32 \\ 1529 & 10 & 0.31 \\ 1793 & 11 & 0.30 \\ 2076 & 12 & 0.29 \\ 2378 & 13 & 0.28 \\ 2699 & 14 & 0.28 \\ 3039 & 15 & 0.27 \\ 3399 & 16 & 0.27 \\ 3778 & 17 & 0.26 \\ 4177 & 18 & 0.26 \\ 4596 & 19 & 0.25 \\ 5036 & 20 & 0.25 \\ 5497 & 21 & 0.24 \\ 5979 & 22 & 0.24 \\ 6483 & 23 & 0.23 \\ 7009 & 24 & 0.23 \\ 7558 & 25 & 0.22 \\ 8130 & 26 & 0.22 \\ 8726 & 27 & 0.22 \\ 9346 & 28 & 0.21 \\ 9991 & 29 & 0.21 \\ 10661 & 30 & 0.21 \\ 11358 & 31 & 0.20 \\ 12082 & 32 & 0.20 \\ 12834 & 33 & 0.20 \\ 13614 & 34 & 0.19 \\ 14424 & 35 & 0.19 \\ 15265 & 36 & 0.19 \\ 16137 & 37 & 0.19 \\ 17042 & 38 & 0.18 \\ 17981 & 39 & 0.18 \\ 18955 & 40 & 0.18\end{array}$




$\begin{array}{lll}19965 & 41 & 0.17 \\ 21013 & 42 & 0.17 \\ 22101 & 43 & 0.17 \\ 23230 & 44 & 0.17 \\ 24402 & 45 & 0.16 \\ 25618 & 46 & 0.16 \\ 26881 & 47 & 0.16 \\ 28193 & 48 & 0.16 \\ 29557 & 49 & 0.15 \\ 30975 & 50 & 0.15 \\ 32450 & 51 & 0.15 \\ 33986 & 52 & 0.15 \\ 35586 & 53 & 0.14 \\ 37253 & 54 & 0.14 \\ 38992 & 55 & 0.14 \\ 40808 & 56 & 0.14 \\ 42707 & 57 & 0.13 \\ 44694 & 58 & 0.13 \\ 46776 & 59 & 0.13 \\ 48961 & 60 & 0.13 \\ 51258 & 61 & 0.13 \\ 53677 & 62 & 0.12 \\ 56230 & 63 & 0.12 \\ 58932 & 64 & 0.12 \\ 61799 & 65 & 0.12 \\ 64851 & 66 & 0.11 \\ 68113 & 67 & 0.11 \\ 71617 & 68 & 0.11 \\ 75401 & 69 & 0.10 \\ 79517 & 70 & 0.10 \\ 84035 & 71 & 0.10 \\ 89053 & 72 & 0.10 \\ 94717 & 73 & 0.09\end{array}$

Table 12: Parameters for Highspeed TCP. 


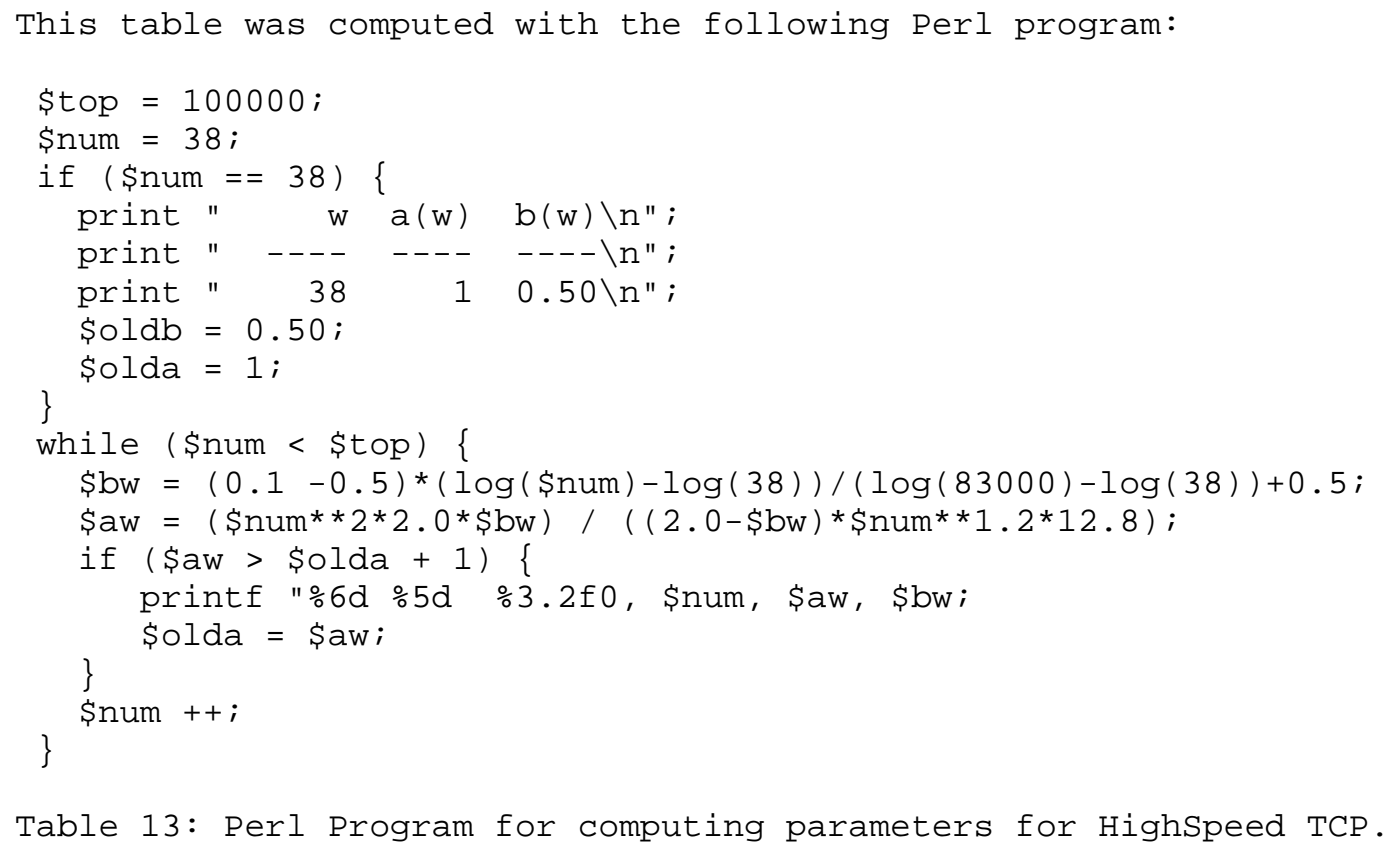


C. Exploring the time to converge to fairness.

This section gives the Perl program used to compute the congestion window growth during congestion avoidance.

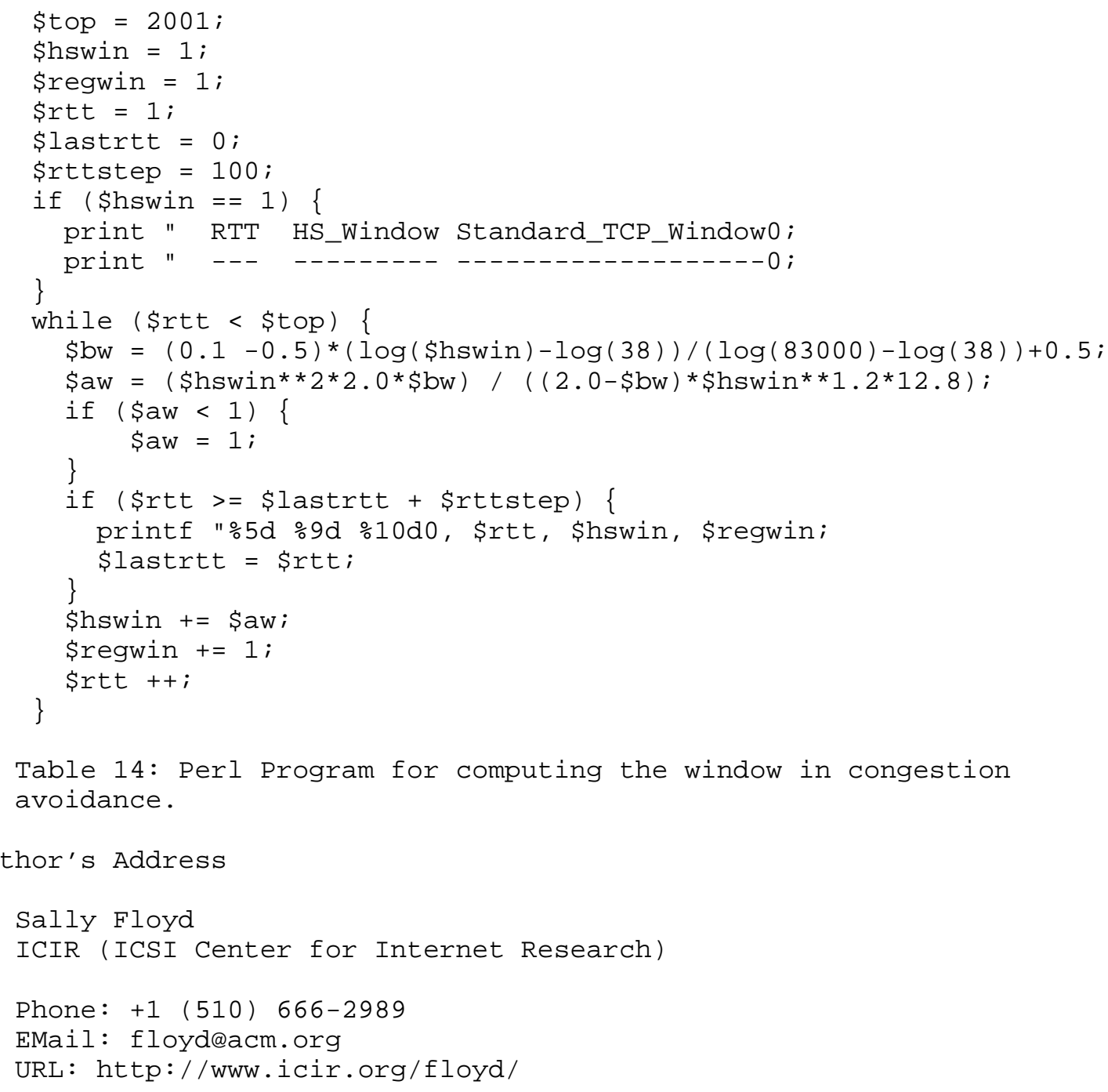


Full Copyright statement

Copyright (C) The Internet Society (2003). All Rights Reserved.

This document and translations of it may be copied and furnished to others, and derivative works that comment on or otherwise explain it or assist in its implementation may be prepared, copied, published and distributed, in whole or in part, without restriction of any kind, provided that the above copyright notice and this paragraph are included on all such copies and derivative works. However, this document itself may not be modified in any way, such as by removing the copyright notice or references to the Internet society or other Internet organizations, except as needed for the purpose of developing Internet standards in which case the procedures for copyrights defined in the Internet Standards process must be followed, or as required to translate it into languages other than English.

The limited permissions granted above are perpetual and will not be revoked by the Internet society or its successors or assignees.

This document and the information contained herein is provided on an "AS IS" basis and THE INTERNET SOCIETY AND THE INTERNET ENGINEERING TASK FORCE DISCLAIMS ALL WARRANTIES, EXPRESS OR IMPLIED, INCLUDING BUT NOT LIMITED TO ANY WARRANTY THAT THE USE OF THE INFORMATION HEREIN WILL NOT INFRINGE ANY RIGHTS OR ANY IMPLIED WARRANTIES OF MERCHANTABILITY OR FITNESS FOR A PARTICULAR PURPOSE.

Acknowledgement

Funding for the RFC Editor function is currently provided by the Internet Society. 